\title{
Frequency Response and Tunability of Grating-Assisted Directional Couplers
}

\author{
Giora Griffel and Amnon Yariv
}

\begin{abstract}
The spectral properties of grating-assisted directional couplers are studied using an improved coupled mode formulation. Key parameters for the design of these structures, such as the grating period, the coupling length, and other structural parameters, are calculated. The frequency response, the filter bandwidth, and the tuning range are analyzed. The technique is used to examine a specific case of InGaAsP-InP tunable filter, and the results are compared to a prior experiment.
\end{abstract}

\section{INTRODUCTION}

$\mathbf{R}$ ECENTLY there has been an extensive research effort [1]-[4] to fabricate broad-band tunable semiconductor laser, with the objective of covering the entire gain-bandwidth, preferably with single longitudinal-mode operation. Such a device would play a major role in spectroscopy, wavelength-division multiplexed systems, as well as in coherent heterodyne-detection systems.

Most of the proposed structures share the feature of being composed of at least two sections, one of which is an active section, responsible for light amplification, while the other serves as a wavelength selective filter whose center wavelength determines the actual lasing wavelength. The common mechanism utilized for that purpose employs the wavelength selectivity of backward coupling which is provided by an etched grating, namely, a distributed Bragg reflector (DBR). A related, recently demonstrated device [5]-[6] makes use of grating-assisted wavelength-selective coupling between two forwardpropagating channel modes of a directional coupler made of two parallel waveguides. In that case, the waveguides are nonsynchronized, i.e., each one supports an optical mode with a different phase velocity. Therefore, these two modes can only couple energy with the assistance of the grating which provides the necessary phase matching. In this paper, we present a study of the spectral properties of the grating-assisted codirectional coupler, and we compare them to those of the contradirectional type. In particular, we shall give expressions for the fractional bandwidth and the tunability of such devices. We shall examine how these properties are influenced by the structures' parameters. Design formulas for the exact determination of the center wavelength of operation and the full-coupling

Manuscript received June 19, 1990; revised January 28, 1991. This work was supported by the National Science Foundation, the U.S. Office of Naval Research, and the U.S. Army Research Office. The work of G. Griffe was supported by a Bantrell Postdoctoral Fellowship at the California Institute of Technology, Pasadena, CA.

The authors are with the Department of Electrical Engineering and Applied Physics, California Institute of Technology, Pasadena, CA 91125.

IEEE Log Number 9244625. interaction length, as well as other device parameters are given. Finally, the formulation given here is used to examine experimental results obtained by Alferness et al. [6].

\section{ANALYSIS}

The structure analyzed is depicted in Fig. 1. It consists of two dissimilar parallel optical waveguides (a) and (b), which can exchange energy due to the existence of a periodic grating located at one of their boundaries. The grating is described by an index perturbation

$$
\begin{aligned}
\Delta n^{2}(x, y, z) & =f(x, y) \Delta n^{2}(z) \\
& =f(x, y) d\left(e^{i(2 \pi / \Lambda) z}+e^{-i(2 \pi / \Lambda) z}\right) .
\end{aligned}
$$

$f(x, y)$ is a window function which describes the spatial location of the grating, while $d$ is the grating "amplitude" given by $d=\left(n_{i}^{2}-n_{j}^{2}\right) / \pi$ where $n_{i, j}$ is related to the layers on both sides of the grating. $\Lambda$ is the grating period, and it is chosen to match the propagation constants $\gamma_{a}$ and $\gamma_{b}$ of the optical modes in each of the waveguides:

$$
\frac{2 \pi}{\Lambda} \cong \gamma_{a}-\gamma_{b} \equiv 2 \Delta \gamma
$$

$\gamma_{a}$ and $\gamma_{b}$ are corrected forms of the the propagation constants of the isolated waveguides, which take the modal interaction into account, namely,

$$
\begin{aligned}
& \gamma_{a}=\beta_{a}+\left(\tilde{K}_{a a}-c \tilde{K}_{b a}\right) /\left(1-c^{2}\right) \\
& \gamma_{b}=\beta_{b}+\left(\tilde{K}_{b b}-c \tilde{K}_{a b}\right) /\left(1-c^{2}\right) .
\end{aligned}
$$

The coefficients $\tilde{K}_{p q}$ are given by [7]

$$
\tilde{K}_{p q}=\frac{\omega}{4} \iint_{-\infty}^{+\infty} \Delta \epsilon^{(q)}\left[E_{t}^{(p)} E_{t}^{(q)}-\frac{\epsilon^{(p)}}{\epsilon} E_{z}^{(p)} E_{z}^{(q)}\right] d x d y
$$

where we have substituted $(p, q)=(a, b) . E_{t}$ and $E_{z}$ are the transverse and longitudinal components of the optical field, respectively. $\epsilon^{(p)}$ and $\epsilon$ are the lateral $(x, y)$ dependency of the dielectric constants of the isolated waveguide $(p)$ and the whole structure, respectively, and $\omega$ is the angular field frequency. The parameter $c$ in (3) is defined by the overlap integral of the two waveguide modes:

$$
c=\frac{1}{2}\left(c_{a b}+c_{b a}\right) ; \quad c_{p q}=\frac{1}{2} \iint_{-\infty}^{+\infty}\left[E_{t}^{(p)} \times H_{t}^{(q)}\right]_{z} d x d y .
$$




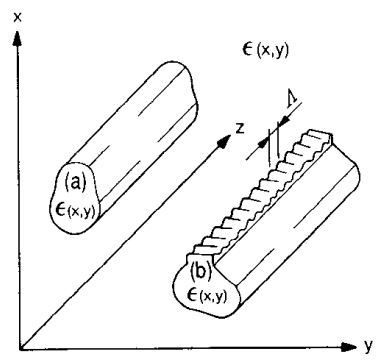

Fig. 1. A general structure of grating-assisted codirectional coupler.

The deviation from exact matching is characterized by the parameter $\delta$ where

$$
2 \delta \equiv 2 \Delta \gamma-\frac{2 \pi}{\Lambda} .
$$

The coupled mode equations for this structure are

$$
\begin{aligned}
& \frac{d}{d z} A(z)=i d k_{a b} e^{-i 2 \delta z} B(z) \\
& \frac{d}{d z} B(z)=i d k_{b a} e^{i 2 \delta z} A(z)
\end{aligned}
$$

where $A(z)$ and $B(z)$ are the slowly varying amplitudes of the optical mode propagating in each of the isolated waveguides $(a)$ and $(b)$, respectively. The coupling coefficients $k_{a b}$ and $k_{b a}$ are given by

$$
\begin{aligned}
& k_{a b}=\left(\hat{K}_{b a}-c \hat{K}_{b b}\right) /\left(1-c^{2}\right) \\
& k_{b a}=\left(\hat{K}_{a b}-c \hat{K}_{a a}\right) /\left(1-c^{2}\right)
\end{aligned}
$$

and

$$
\hat{K}_{p q}=\frac{\omega}{4} \epsilon_{0} \iint_{-\infty}^{+\infty} f(x, y)\left[E_{t}^{(p)} E_{t}^{(q)}-\frac{\epsilon^{(p)}}{\epsilon} E_{z}^{(p)} E_{z}^{(q)}\right] d x d y .
$$

An extensive study of the coupled-mode equation (7) was carried out in [7]. Here, we would like to address the issue of how the coupling efficiency and other structural parameters such as the interaction length and the grating period are changed as a function of the optical wavelength. If the boundary conditions are such that energy is coupled into waveguide $(a)$ at $z=0$, then the fraction of power coupled to waveguide $(b)$ at a distance $z=L$ is given by [7]

$$
\eta(z) \cong c_{a b} c_{b a}+\frac{1-c_{a b} c_{b a}}{1+\zeta^{2}} \sin ^{2}\left[d \sqrt{k_{a b} k_{b a}}\left(1+\zeta^{2}\right)^{1 / 2} z\right]
$$

where $\zeta$ is a normalized detuning factor defined by

$$
\zeta \equiv \frac{\delta}{d \sqrt{k_{a b} k_{b a}}} .
$$

The expression for coupling efficiency, given by (10), is almost accurate for most practical cases. The conditions for the validity of this approximation are detailed in [7]. The maximum efficiency is obtained at a distance $l$ for which the $\sin$ argument equals $\pi / 2$, and is therefore given by

$$
l_{\max }=\frac{\pi}{2 d \sqrt{k_{a b} k_{b a}}\left(1+\zeta^{2}\right)^{1 / 2}} .
$$

The efficiency curve as a function of the wavelength around the phase-matching point $(\zeta=0)$ was calculated for a specific case with a device length $z=l_{\max }$ and is shown in Fig. 4. It is seen that the device functions as an optical filter, owing its frequency selectivity to the strong dependency of the parameter $\zeta$ on the frequency of operation. For that case, assuming $c_{a b} c_{b a} \approx 0$, the bandwidth of the filter can be calculated using the value of $\zeta$ for which the efficiency drops to half its maximum value, $\zeta_{1 / 2}=$ \pm 0.799 . This value is substituted into (6) and (11), where we use $\Delta \gamma=\gamma_{a}-\gamma_{b} \equiv k_{0}\left(n_{\text {eff }_{b}}-n_{\text {eff }_{a}}\right)$ with $n_{\text {eff }_{a . b}}$ approximated near the phase matching wavelength $\lambda_{0}$ by

$$
n_{\mathrm{eff}_{a, b}(\lambda)}=n_{\mathrm{eff}_{a, b\left(\lambda_{0}\right)}}+\frac{d}{d \lambda} n_{\operatorname{eff}_{a, b}\left(\lambda_{0}\right)}\left(\lambda-\lambda_{0}\right)
$$

where $n_{\mathrm{eff}_{a, b}} \equiv \gamma_{a, b} / k_{0}, k_{0}$ being the vacuum propagation constant. Using the outlined derivation, the fractional filter bandwidth is found to be

$$
\frac{\Delta \lambda}{\lambda_{0}}=\zeta_{1 / 2} \frac{\Lambda}{l} \frac{1}{1-\Lambda \frac{d}{d \lambda}\left(n_{\mathrm{eff} b}-n_{\mathrm{eff}_{a}}\right)_{\left(\lambda_{0}\right)}} .
$$

An expression for the tunability of the filter can be obtained in a similar manner. If we assume that due to some externally applied mechanism, such as carrier injection or electrooptical effect, the magnitude and the slope of the dispersion curve of each of the waveguides is changed by the amounts $\Delta n_{\text {eff }}$ and $\Delta(d / d \lambda) n_{\text {eff }}$, that is,

$$
\begin{aligned}
n_{\mathrm{eff}_{a, b}(\lambda)=} & n_{\mathrm{eff}_{a, b}\left(\lambda_{0}\right)}+\Delta n_{\mathrm{eff}_{a, b}\left(\lambda_{0}\right)}+\frac{d}{d \lambda} n_{\mathrm{eff}_{a, b\left(\lambda_{0}\right)}\left(\lambda-\lambda_{0}\right)} \\
& +\Delta \frac{d}{d \lambda} n_{\mathrm{eff}_{a, b}\left(\lambda_{0}\right)}\left(\lambda-\lambda_{0}\right)
\end{aligned}
$$

then the fractional shift of the center wavelength of the filter curve is given by

$$
\begin{aligned}
\frac{\Delta \lambda_{0}}{\lambda_{0}}= & \frac{\Delta\left[n_{\mathrm{eff}_{b}}-n_{\mathrm{eff}_{a}}\right]_{\left(\lambda_{0}\right)}}{\left[n_{\mathrm{eff}_{b}}-n_{\left.\mathrm{eff}_{a}\right]_{\left(\lambda_{0}\right)}}\right.} \\
& \cdot \frac{1}{1-\Lambda\left[\left(\frac{d}{d \lambda}+\Delta \frac{d}{d \lambda}\right)\left(n_{\mathrm{eff}_{b}}-n_{\mathrm{eff}_{a}}\right)_{\left(\lambda_{0}\right)}\right)}
\end{aligned}
$$

or

$$
\frac{\Delta \lambda_{0}}{\lambda_{0}}=\frac{\Lambda}{\lambda_{0}} \frac{\Delta\left[n_{\mathrm{eff}_{b}}-n_{\mathrm{eff}_{a}}\right]_{\left(\lambda_{0}\right)}}{1-\Lambda\left[\left(\frac{d}{d \lambda}+\Delta \frac{d}{d \lambda}\right)\left(n_{\mathrm{eff} b}-n_{\mathrm{eff}_{a}}\right)_{\left(\lambda_{0}\right)}\right]} .
$$

Repeating the same calculation for the case of a singlewaveguide DBR structure results in a similar expression for the fractional center wavelength shift, except that $n_{\text {eff }}$ of the only waveguide of such a device appears instead of 

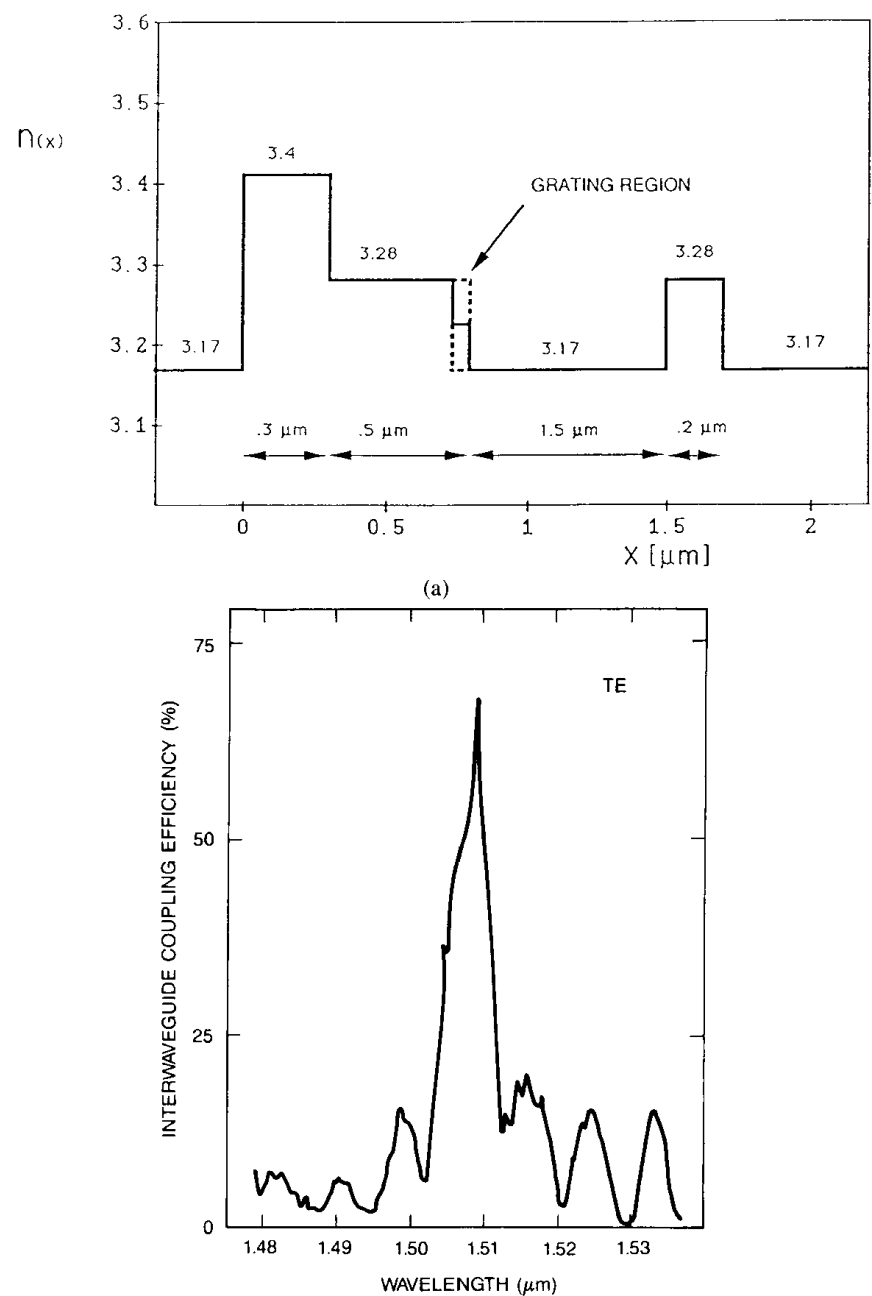

(b)

Fig. 2. (a) A schematic drawing of the grating-assisted codirectional slab coupler made of InGaAsP-InP. (b) Measured coupling efficiency versus wavelenth. (After Alferness et al. [6].)

the effective refractive index difference of the two coupled waveguides in the present case. This difference in the denominator of the first term on the right side of (16) is the reason for the much higher tuning range of the codirectional coupler compared to the contradirectional one. This change manifests itself by the grating period $\Lambda$ which appears in the numerator on the right side of (17), so that as a rule of thumb, one can say that the fractional tunability is improved by a factor equal to the ratio of the grating periods of the two cases. It should be noted that by having a much longer grating period, the fractional bandwidth might increase as well, but this feature can be retained by increasing the interaction length, which appears in the denominator of the expression. To achieve that, we can simply decrease the coupling coefficient by either increasing the waveguide's separation or reducing the grating depth.

\section{Application Example}

We applied the above formalism to the case of a grating-assisted directional coupler made of InGaAsP-InP, which was fabricated and presented by Alferness et al. [6]. In that case, a variable period grating was etched between two slab waveguides made of vertically stacked InGaAsP-InP layers. A schematic drawing of the device is shown in Fig. 2. Using a simple modal analysis in order to match the propagation constants of the isolated waveguides, for a filter with a center wavelength of $\lambda_{0}=1.5$ $\mu \mathrm{m}$, results in a grating period of $\Lambda=14.5 \mu \mathrm{m}$. However, it was found in the experiment that in the actual device, there was a shift of the center wavelength towards a higher value, and a center wavelength of $\lambda_{0}=1.51 \mu \mathrm{m}$ was observed for a grating period of $\Lambda=14.4 \mu \mathrm{m}$. The device length was $1 \mathrm{~mm}$ and the grating thickness was $0.06 \mu \mathrm{m}$.

We have used (2) and (12) in order to calculate the grating period and the full coupling length as a function of the desired wavelength of the filter transfer curve. The material dispersion for this calculation as well as in the rest of the paper was taken into consideration by using a two-oscillator model for the calculation of the refractive indexes of the structure [8]. The results are shown in Fig. 


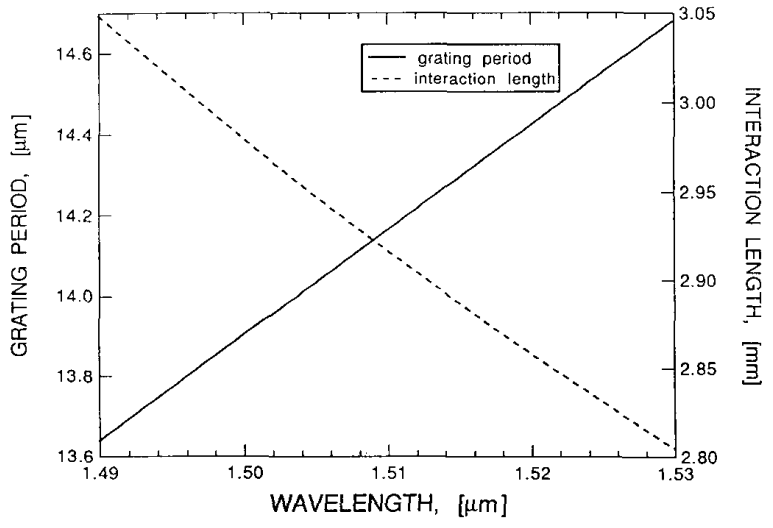

Fig. 3. The grating period (left $Y$ axis) and the full coupling length (right $Y$ axis, dashed line) as function of the desired center wavelength of the filter.

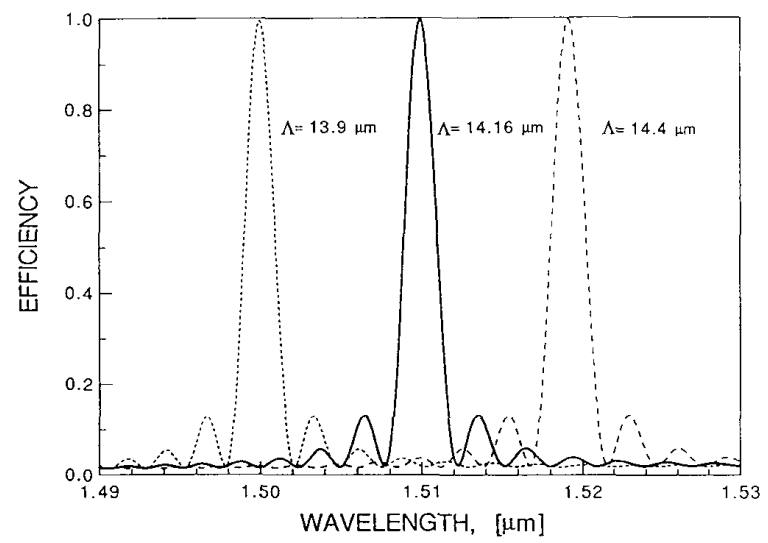

Fig. 4. The calculated coupling efficiency for the grating-assisted codirec tional coupler of [6] for three different grating periods.

3. An almost-linear dependency of the grating period on the center wavelength and an inverse proportionality of the full interaction length is clearly observed. It was found, using the present formulation, that in order to obtain a center wavelength of $\lambda_{0}=1.5 \mu \mathrm{m}$, a grating period of $\Lambda=13.9 \mu \mathrm{m}$ should be used, while an increase of the grating period would lead to a higher value of the center frequency; a grating period of $\Lambda=14.16 \mu \mathrm{m}$ would lead to a center wavelength of $\lambda_{0}=1.51 \mu \mathrm{m}$, and a grating period of $\Lambda=14.4 \mu \mathrm{m}$, which is the one used in the discussed experiment, should result in a center wavelength of $\lambda_{0}=1.519 \mu \mathrm{m}$. The calculated full coupling length of the device is in the range of $2.8-3 \mathrm{~mm}$. This fact can explain why the maximum efficiency in the experimental device, whose length was only $1 \mathrm{~mm}$, is on the order of $65 \%$, and its bandwidth is about three times the calculated value. These results are in excellent agreement with the compared experiment.

The coupling efficiency for the three mentioned grating periods was calculated using (10), and the results are shown in Fig. 4. As expected, the bandwidth shown by these curves is much narrower and fits the value calculated (14) for a full coupling length. One way to increase the coupling efficiency of the tested device could be to

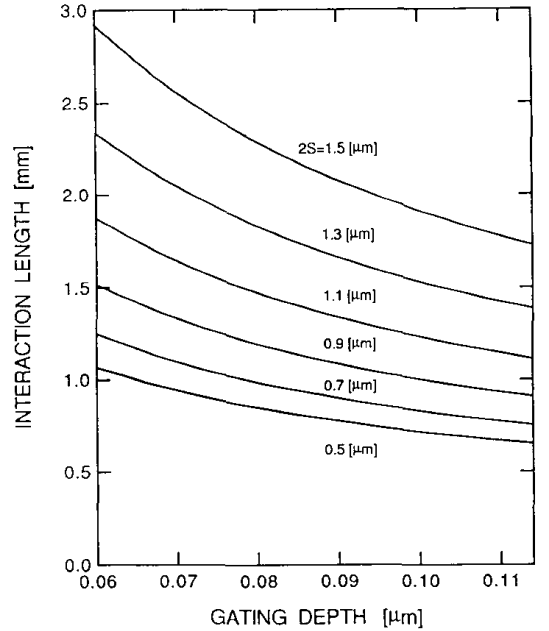

Fig. 5. The calculated full coupling length as a function of the grating depth; the waveguide separation $2 s$ is used as a parameter

reduce the full coupling length to the practical value of 1 $\mathrm{mm}$. This can be done by increasing the coupling coefficient through either etching a deeper grating or reducing the distance between the waveguides. A calculation of the full coupling length as a function of the grating depth and with a waveguide separation of $2 s$ used as a parameter is shown in Fig. 5, from which device parameters for optimum performance can be obtained.

\section{CONCLUSIONS}

We have presented a formulation for analyzing and designing grating-assisted directional coupler filters. The formalism enables us to obtain desired filter characteristics, such as center wavelength, bandwidth, and tunability. An application example was examined and found to be in agreement with experimental results.

\section{REFERENCES}

[1] Y. Yoshikuni, K. Oe, G. Motosugi, and T. Matsuoka, "Broad wave length tuning under single-mode oscillation with a multi-electrode distributed feedback laser," Electron. Lett., vol. 22, pp. 1153-1154, Oct. 1986.

[2] N. K. Dutta, A. B. Piccirilli, T. Cella, and R. L. Brown, "Electronically tunable distributed feedback laser," Appl. Phys. Lett., vol. 48, pp. 1501-1503, June 1986.

[3] Y. Kotaki, M. Matsuda, H. Ishikawa, and H. Imai, "Tunable DBR laser with wide tuning range,"' Electron. Lett., vol. 24, pp. 503-505, 1988

[4] S. Illek, W. Thulke, C. Schanen, H. Lang, and M. C. Amann, "Over $7 \mathrm{~nm}(875 \mathrm{GHz})$ continuous wavelength tuning by tunable twin-guide (TTG) laser diode," Electron. Lett., vol. 26, pp. 46-47, Jan. 1990.

[5] T. L. Koch, E. G. Burkhardt, F. G. Storz, T. J. Bridges, and T Sizer, "Vertically grating-coupled ARROW structures for III-V integrated optics," IEEE J. Quantum Electron., vol. QE-23, pp. 889897 , June 1987

[6] R. C. Alferness, T. L. Koch, L. L. Buhl, F. Storz, F. Heismann, and M. J. R. Martyak, "Grating-assisted InGaAsP/InP vertical codirectional coupler filter," Appl. Phys. Lett., vol. 55, pp. 2011-2013, Nov. 1989.

[7] G. Griffel, M. Itzkovitch, and A. Hardy, "Coupled mode formulation for directional couplers with longitudinal perturbation," IEEE $J$. Quantum Electron., to be published.

[8] C. H. Henry, L. F. Johnson, R. A. Logan, and D. P. Clarke, “Determination of the refractive index of InGaAsP epitaxial layers by mode line luminescence spectroscopy," IEEE J. Quantum Electron. vol. QE-21, pp. 1887-1985, Dec. 1985 